\title{
Vinen turbulence via the decay of multicharged vortices in trapped atomic Bose-Einstein condensates
}

\author{
A. Cidrim,,${ }^{1,}$ A. C. White, ${ }^{2}$ A. J. Allen, ${ }^{3}$ V. S. Bagnato, ${ }^{1}$ and C. F. Barenghi ${ }^{3}$ \\ ${ }^{1}$ Instituto de Física de São Carlos, Universidade de São Paulo, C.P. 369, 13560-970 São Carlos, SP, Brazil \\ ${ }^{2}$ Quantum Systems Unit, Okinawa Institute of Science and Technology, Okinawa 904-0495, Japan \\ ${ }^{3} J o i n t$ Quantum Centre (JQC) Durham-Newcastle, School of Mathematics and Statistics, Newcastle University, \\ Newcastle upon Tyne NE1 7RU, United Kingdom \\ (Received 22 April 2017; published 21 August 2017)
}

\begin{abstract}
We investigate a procedure to generate turbulence in a trapped Bose-Einstein condensate which takes advantage of the decay of multicharged vortices to reduce surface oscillations. We show that the resulting singly charged vortices twist around each other, intertwined in the shape of helical Kelvin waves, which collide and undergo vortex reconnections, creating a disordered vortex state. By examining the velocity statistics, the energy spectrum, the correlation functions, and the temporal decay and comparing these properties with the properties of classical turbulence and observations in superfluid helium, we conclude that this disordered vortex state can be identified with the Vinen regime of turbulence which has been discovered in the context of superfluid helium.
\end{abstract}

DOI: 10.1103/PhysRevA.96.023617

\section{MOTIVATION}

The singular nature of quantized vorticity (concentrated along vortex lines) and the absence of viscosity make superfluids remarkably different from classical fluids. Nevertheless, recent studies [1] have revealed that superfluid helium, when suitably stirred, shares an important property with classical turbulence: the same Kolmogorov energy spectrum [2], describing a distribution of kinetic energy over the length scales which signifies an energy cascade from large length scales to small length scales. This finding suggests that the turbulence of quantized vortices (quantum turbulence) may represent the 'skeleton' of classical turbulence [3].

A puzzle arises, however: experiments [4,5] show that, besides Kolmogorov (or quasiclassical) turbulence, there is another regime in which turbulent superfluid helium lacks the Kolmogorov spectrum: Vinen turbulence (also known as the 'ultraquantum regime'). In their experiments with superfluid helium, Walmsley and Golov [4] were able to generate both regimes by controlling injections of vortex rings in their system. When monitoring the temporal decay of the vortex line density $L(t)$, they observed that short injections of vortex rings produced a regime in which $L(t) \sim t^{-1}$, as opposed to long injections, which displayed the scaling $L(t) \sim t^{-3 / 2}$. Numerical simulations [6] of this experiment revealed that Vinen turbulence [decaying as $L(t) \sim t^{-1}$ ] has a spectrum $E(k)$ which peaks at intermediate length scales and behaves as $E(k) \sim k^{-1}$ for large $k$ in the hydrodynamical range $k<2 \pi / \ell$ (where $\ell$ is the average intervortex distance); on the contrary, Kolmogorov turbulence [decaying as $L(t) \sim t^{-3 / 2}$ ] has a spectrum $E(k)$ which peaks at the largest length scales and behaves as $E(k) \sim k^{-5 / 3}$ for large $k$.

\footnotetext{
*andrecidrim@gmail.com

Published by the American Physical Society under the terms of the Creative Commons Attribution 4.0 International license. Further distribution of this work must maintain attribution to the author(s) and the published article's title, journal citation, and DOI.
}

It is now understood that Vinen's pioneering experiments [7] on counterflow heat transfer in superfluid helium also generated Vinen turbulence. This was demonstrated by numerical models of counterflow turbulence [8] driven by a uniform normal fluid which produced a superfluid energy spectrum peaking at mesoscales with the expected $E(k) \sim k^{-1}$ tail at large $k$. Additionally, the $L(t) \sim t^{-1}$ vortex line density decay was also observed, which is the large- $t$ decaying solution of the equation $d L / d t \sim-L^{2}$ proposed by Vinen on simple physical arguments to model a randomlike flow. Moreover, a recent work [9] examining the properties of turbulence following the thermal quench of a Bose gas has found that topological defects created by the Kibble-Zurek mechanism evolve into a turbulent vortex tangle [10] which eventually decays into a vortex-free state. During the decay, which has the form $L(t) \sim t^{-1}$, the energy spectrum is concentrated at intermediate wave numbers and scales as $E(k) \sim k^{-1}$, and the velocity correlation functions drop to a few percent over the distance $\ell$. The thermal quench is therefore a third clear example of Vinen turbulence. It has been argued [5] that, physically, Vinen turbulence differs from Kolmogorov turbulence because it lacks the energy cascade from large to small eddies which is characteristic of classical turbulence. At the moment, it is not clear whether this distinction between Vinen turbulence and Kolmogorov turbulence, first proposed by Volovik [11], is unique to superfluids. It is in principle possible that the former regime can appear only in systems with discrete vorticity, ruling out its presence in classical fluids (in which vorticity is a continuous quantity).

Given this context, it is clear that trapped atomic BoseEinstein condensates (BECs) are ideal systems to tackle this puzzle and to eventually serve as a test bed for a crossover between Vinen and Kolmogorov turbulence. This is because, unlike helium, the physical properties of BECs (e.g., the strength of atomic interactions, the density, the vortex core radius) can be controlled. Moreover, individual quantized vortices are more easily nucleated, manipulated $[12,13]$, and observed [14-16] in BECs than in helium.

The second motivation behind our work is that the study of three-dimensional (3D) turbulence in BECs [17] is held back 
by the lack of a standard method to excite turbulence in a reproducible way, so that experiments and numerical simulations can be compared with each other and any generality of results can be more easily recognized. In classical turbulence, standard benchmarks are flows driven along channels or stirred by propellers, flows around well-defined obstacles (e.g. cylinders, spheres, steps), and wind tunnel flows past grids. Similar techniques are used for superfluid helium [18,19], which is mechanically or thermally driven along channels or stirred by oscillating wires, grids, forks, propellers, and spheres. Vortices and turbulence in BECs have been generated, either numerically or experimentally, by moving a laser beam across the BEC [15,20-26], by shaking [27] or stirring the trap, rotating it around two perpendicular axes [28], by phase imprinting staggered vortices [29], or by thermally quenching the system (Kibble-Zurek mechanism) [30-33]. This variety of techniques and the arbitrarily chosen values of physical parameters mean that comparisons are difficult. Moreover, the disadvantage of some of these techniques is that they tend to induce large surface oscillations or even fragmentation [34] of the condensate, which complicates the interpretation of results and the comparison with classical turbulence.

The aim of this report is to propose a technique to induce turbulence in BECs based on the decay of multicharged vortices (a more controlled and less forceful technique than the above-mentioned methods) and to characterize the turbulence which is produced. For this purpose, we compare two disordered vortex states, which we call "anisotropic" and "quasi-isotropic" for simplicity, resulting from the decay of a single $j=4$ charged vortex and from the decay of two antiparallel $j=2$ vortices, respectively. We reveal a connection between BEC turbulence and the Vinen turbulent regime discovered in superfluid helium.

\section{MULTICHARGED VORTICES}

In a superfluid, the circulation around a vortex line is an integer multiple $(j=1,2, \ldots)$ of the quantum of circulation [35] $\kappa=h / m$, that is, $\oint_{C} \mathbf{v} \cdot d \ell=j \kappa$, where $C$ is a closed path around the vortex line, $h$ is Planck's constant, and $m$ the atomic mass. The velocity field around an isolated vortex line is therefore constrained to the form $v=j \kappa /(2 \pi r)$, where $r$ is the distance to the vortex axis. This property is in marked contrast to classical fluids, where the velocity of rotation about an axis (e.g., swirls, tornadoes, galaxies) has arbitrary strength and radial dependence.

The angular momentum and the energy of an isolated vortex in a homogeneous superfluid grow, respectively, with $j$ and $j^{2}$ [35]. Therefore, for the same angular momentum, multicharged $(j>1)$ vortices carry more energy and, in the presence of thermal dissipative mechanisms, tend to decay into singly charged vortices [36-39]. Besides the energy instability, there is also a dynamical instability [40-42], which would destabilize a multicharged vortex. The time scale of these effects has been investigated [36,38,39,41,43-46]. The technique of topological phase imprinting [47] has allowed the controlled generation of multicharged vortices [36,38,45,48] in atomic condensates. The decay of a doubly quantized vortex into two singly quantized vortices has been studied $[36,43,44]$ in a Na BEC. Quadruply charged quantized vortices are also theoretically predicted [49] to decay. Recent work has determined that the stability of such vortices is affected by the condensate's density [36] and size [44] and by the nature of the perturbations [49].

\section{MODEL}

We model the condensate's dynamics using the 3D GrossPitaevskii equation (GPE) [35] for a zero-temperature condensate,

$$
i \hbar \frac{\partial \psi}{\partial t}=\left(-\frac{\hbar^{2}}{2 m} \nabla^{2}+U(\mathbf{r})+g|\psi|^{2}\right) \psi,
$$

where $\psi(\mathbf{r}, t)$ is the condensate's wave function, $\mathbf{r}$ the position, $t$ the time, and

$$
U(\mathbf{r})=\frac{m}{2}\left(\omega_{x}^{2} x^{2}+\omega_{y}^{2} y^{2}+\omega_{z}^{2} z^{2}\right)=\frac{m}{2}\left(\omega_{r} r^{2}+\omega_{z} z^{2}\right)
$$

the harmonic trapping potential. The parameter $g=$ $4 \pi \hbar^{2} a_{s} / m$ characterizes the strength of the interatomic interactions, where $a_{s}$ is the $s$-wave scattering length. The normalization is $\int_{V}|\psi|^{2} d V=N$, where $V$ is the BEC's volume and $N$ the number of atoms. We cast the GPE in dimensionless form using $\tau_{\mathrm{HO}}=\omega_{r}^{-1}, \ell_{\mathrm{HO}}=\sqrt{\hbar / m \omega_{r}}$, and $\hbar \omega_{r}$ as units of time, distance, and energy, respectively. The interatomic interaction parameter, $g(=8600)$, is chosen to describe a typical BEC with $N \approx 1 \times 10^{5}$ atoms of ${ }^{87} \mathrm{Rb}$ trapped harmonically in a cigar-shaped BEC with radial and axial frequencies such that $\omega_{z} / \omega_{r}=\lambda=0.129$. It is of our particular interest to study properties of the condensate's velocity-field components, which are computed from the definition $\mathbf{v}(\mathbf{r})=\left(\psi \nabla \psi^{*}-\psi^{*} \nabla \psi\right) / 2 i|\psi|^{2}$. The dimensionless GPE is solved numerically in the $3 \mathrm{D}$ domain $-10 \ell_{\mathrm{HO}} \leqslant x, y \leqslant$ $10 \ell_{\mathrm{HO}}$ and $-40 \ell_{\mathrm{HO}} \leqslant z \leqslant 40 \ell_{\mathrm{HO}}$ on a $128 \times 128 \times 512$ grid (keeping the same spatial discretization in the three directions) with time step $\Delta t=10^{-3}$ using the fourth-order RungeKutta method with XMDS2 (eXtensible Multi-Dimensional Simulator 2) [50]. We have performed tests with different grid sizes and verified that our results are independent of the discretization.

\section{DECAY OF SINGLE QUADRUPLY CHARGED VORTEX}

The shape of the singly charged vortex lines emerging from the decay of a multicharged vortex depends on where and when the decay starts. The singly charged lines may be straight or intertwined (as reported here), depending on the perturbation's symmetry and the local density homogeneity. If the perturbation is uniform and the density does not vary much in the $z$ direction, every point on the vortex unwinds at the same rate, and straight singly charged vortex lines will emerge. However, if the density changes significantly along $z$, the unwinding takes place at different times and different positions, inducing intertwining, as discussed in [43].

The twisted vortex decay is shown in Fig. 1 and in the first movie in the Supplemental Material [51]. The main feature visible during the evolution is Kelvin waves. Kelvin waves consist of helical displacements of the vortex core axis and play an important role in quantum turbulence [18]; they have recently been experimentally identified in superfluid helium 


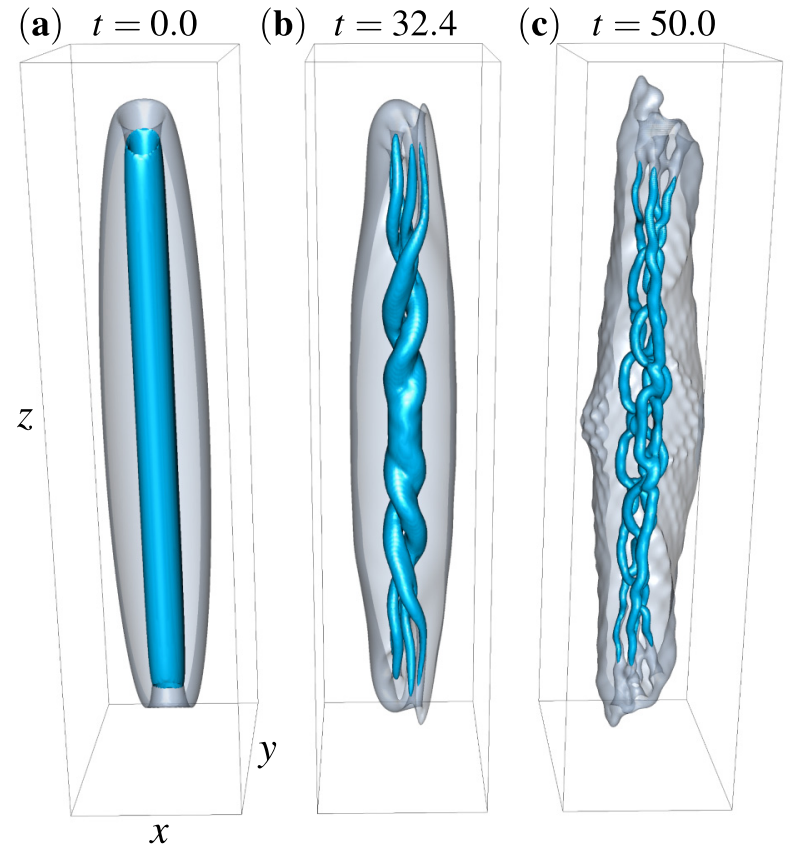

FIG. 1. Three-dimensional isodensity plots show the time (in units of $\tau_{\mathrm{HO}}$ ) evolution of an initial $j=4$ multicharged vortex (a); note the twisted unwinding of the vortex (b), which finally decays into four singly charged $(j=1)$ vortices (c) in the disordered, anisotropic state. The density isosurfaces representing the condensate and the vortices are gray and blue, respectively. The 3D domain is displayed within the plot limits $-7 \ell_{\mathrm{HO}} \leqslant x \leqslant 7 \ell_{\mathrm{HO}},-10 \ell_{\mathrm{HO}} \leqslant y \leqslant 10 \ell_{\mathrm{HO}}$, and $-30 \ell_{\mathrm{HO}} \leqslant z \leqslant 30 \ell_{\mathrm{HO}}$.

[52] and their presence has been recognized in atomic condensates $[53,54]$. Considering Fig. 1, it is worth distinguishing the Kelvin waves which, in our case, emerge on parallel vortices from the decay of a multicharged vortex $[41,44]$ in a confined geometry from the Kelvin waves generated by the Crow instability [55] on antiparallel vortices in a homogeneous condensate.

Despite its known dynamical instability, in the absence of large perturbations, a symmetric multicharged vortex can be a long-lived state in a harmonic trap. We have, for instance, simulated the time evolution for the quadruply charged vortex when symmetrically imprinted along the $z$ direction (i.e., centered at the origin) up to $t=150 \tau_{\mathrm{HO}}$ without verifying its decay (observing instead the development of only lowamplitude collective modes). However, our numerical experiments suggest that the decay of the multicharged vortex can be sped up. Imposing uniformly distributed random fluctuations ( $\leqslant 10 \%$ of $|\psi|$ ) on the initial $j=4$ wave function does not significantly change the decay time scale, probably because the symmetry of the initial condition is not completely broken. A small displacement of the vortex core axis $\left(\approx 0.2 \ell_{\mathrm{HO}}\right)$ is more efficient, triggering the onset of twisted unwinding in about $14.0 \tau_{\mathrm{HO}}$; a larger displacement $\left(\approx 0.5 \ell_{\mathrm{HO}}\right)$ reduces this time to $12.0 \tau_{\mathrm{HO}}$. Among the other methods which we have investigated, the most efficient is to gently squeeze the harmonic potential in the $x y$ plane by an amount $\omega_{x} / \omega_{y}=0.9$ when preparing the initial state in imaginary time, then reset $\omega_{x} / \omega_{y}=1$ when propagating the GPE in real time; the

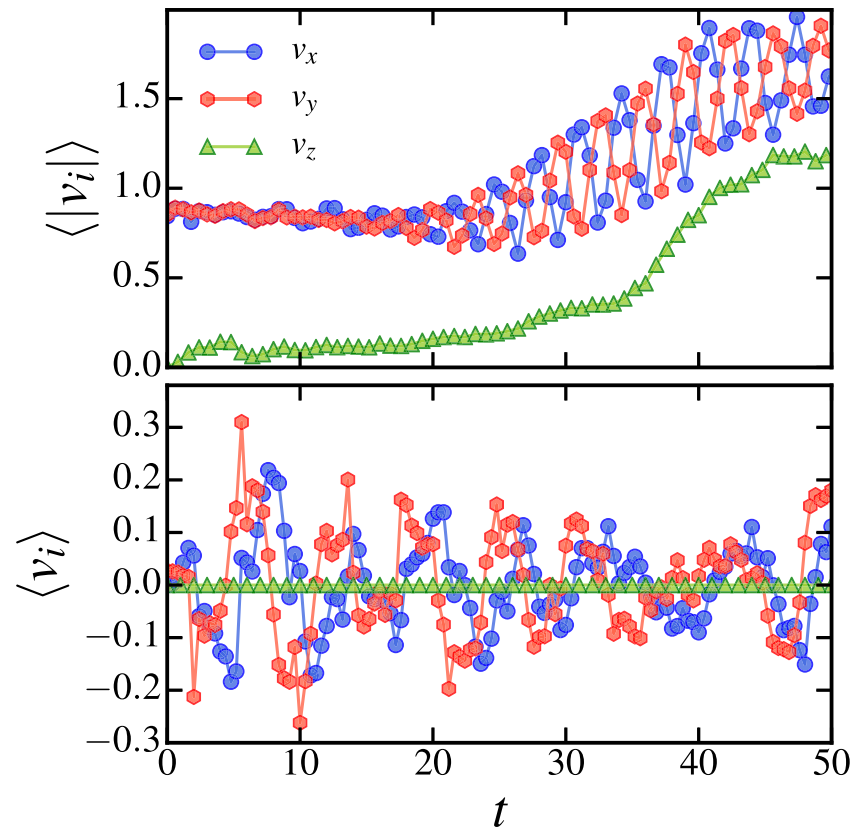

FIG. 2. Decay of a quadruply charged vortex (anisotropic state). Time evolution of average velocity components $v_{i}$ and $\left|v_{i}\right|$ (in units of $\ell_{h o} / \tau_{\mathrm{HO}}$, where $i=x, y, z$ ) vs time (in units of $\tau_{\mathrm{HO}}$ ). The symbol $\langle\ldots\rangle$ denotes the spatial average over the condensate.

squeeze triggers the onset of decay in only $6.0 \tau_{\mathrm{HO}}$. In the experiments, it is usually difficult to control perturbations well enough to reproducibly determine the time scale of decay.

Figure 2 shows the time evolution of the (spatially) averaged velocity components and their magnitudes during the decay. The $x$ and $y$ components display oscillations which become large for $t>20 \tau_{\mathrm{HO}}$, after the initial multicharged vortex has split. The $z$ component behaves differently because all vortices are aligned in the $z$ direction. The oscillations of the transverse velocity components $v_{x}$ and $v_{y}$ appear practically out of phase [see Fig. 2 (top)], and these suggest the existence of collective modes. In order to properly identify these modes we have evaluated the time evolution of the condensate's transverse widths $w_{x}$ and $w_{y}$ (found by adjusting Gaussian fits in the $x$ and $y$ directions over the $z$-integrated density). As shown in Fig. 3, the condensate exhibits a quadrupolar mode, in which $w_{x}$ and $w_{y}$ oscillate out of phase in time. After performing a Fourier analysis of these quadrupolar oscillations we verified the mode's frequency to be $\omega \sim \sqrt{2} \omega_{r}$, in agreement with theoretical predictions for a vortex-free cloud [56]. This mode is reminiscent of the unstable (quadrupolar) Bogoliubov mode that drives the decay of the initial multicharged vortex, as identified for analogous 2D [46] and 3D [42] trapped systems.

For simplicity of reference, we call the resulting disordered vortex configuration an 'anisotropic state'.

\section{DECAY OF TWO ANTIPARALLEL DOUBLY CHARGED VORTICES}

Now we exploit the twisted unwinding of a multicharged vortex as a convenient technique to generate turbulent vortex tangles which are relatively free of high-density perturbations. We start by numerically imprinting antiparallel, doubly 


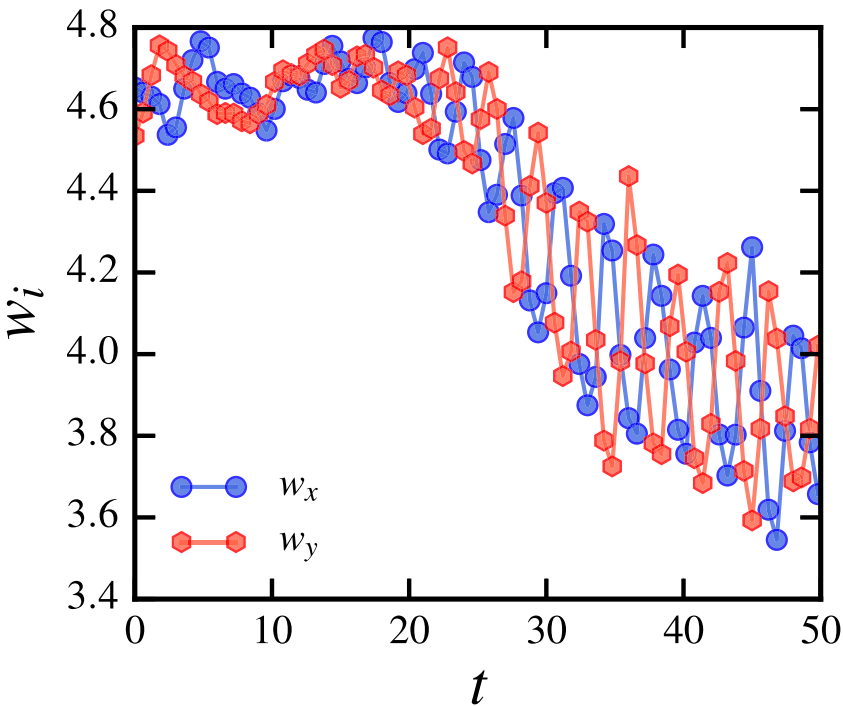

FIG. 3. Decay of quadruply charged vortex (anisotropic state). Time (in units of $\tau_{\mathrm{HO}}$ ) evolution of the condensate's width $w_{i}$ (in units of $\ell_{\mathrm{HO}}$ ).

charged vortices as the initial state. One vortex is centered at position $(x, y)=\left(1.8 \ell_{\mathrm{HO}}, 1.5 \ell_{\mathrm{HO}}\right)$ and the other at $(x, y)=$ $\left(1.0 \ell_{\mathrm{HO}},-1.3 \ell_{\mathrm{HO}}\right)$, as shown in Fig. 4(a). We have performed several simulations, with different initial positions for the vortex pair. When the system was prepared in a symmetric initial state, the vortex pair annihilation was too quick, and less vortex tangling was observed. Since our aim was to induce

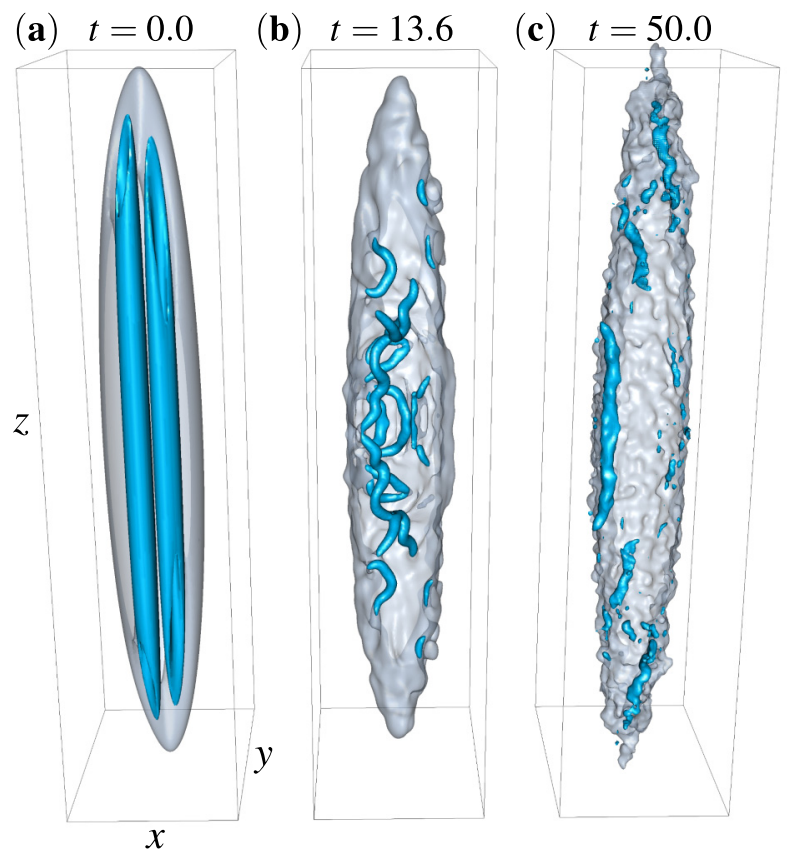

FIG. 4. Isodensity plots showing the evolution (with time $t$ in units of $\left.\tau_{\mathrm{HO}}\right)$ of two initial doubly charged $(j=2)$ antiparallel vortices at $t=0 \tau_{\mathrm{HO}}$ (a) into the turbulent quasi-isotropic state at $t=13.6 \tau_{\mathrm{HO}}$ (b), which has finally decayed at $t=50 \tau_{\mathrm{HO}}$ (c). The $3 \mathrm{D}$ domain is displayed within the plot limits $-7 \ell_{\mathrm{HO}} \leqslant x \leqslant 7 \ell_{\mathrm{HO}},-10 \ell_{\mathrm{HO}} \leqslant y \leqslant$ $10 \ell_{\mathrm{HO}}$, and $-30 \ell_{\mathrm{HO}} \leqslant z \leqslant 30 \ell_{\mathrm{HO}}$.

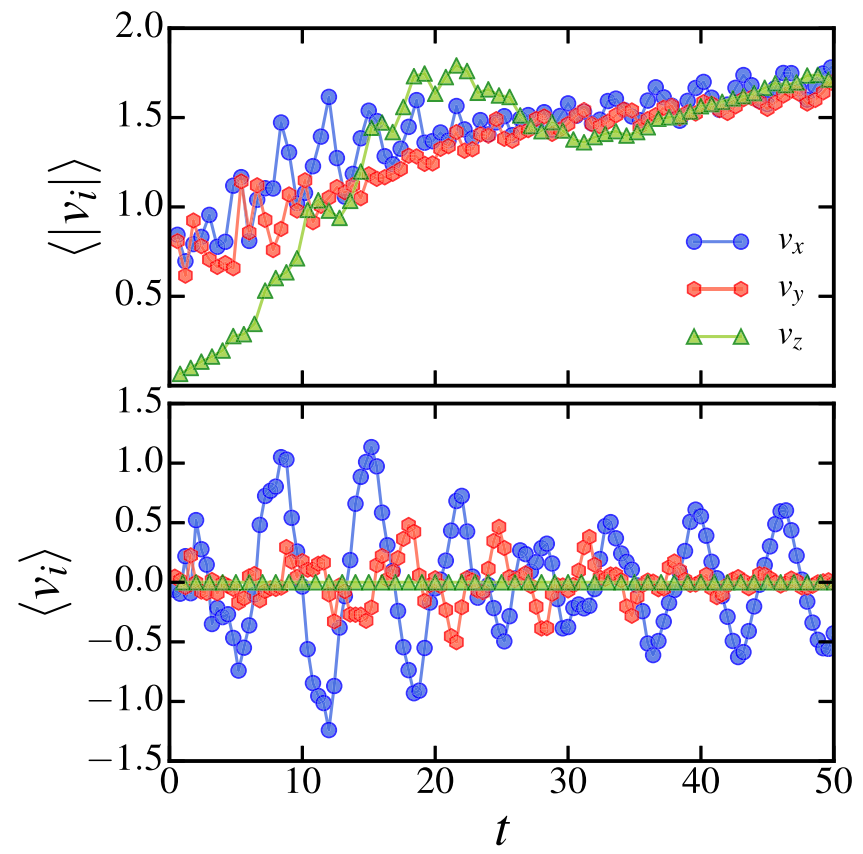

FIG. 5. Decay of antiparallel doubly charged vortices (quasiisotropic state). Time evolution of velocity components as in Fig. 2.

more vortex tangling (therefore stretching the turbulence time window), the values we present are the results of an optimized choice, which breaks the symmetry by imposing a noncentered initial state. During the evolution, the vortices unwind, twist, move slightly forward due to the self-induced velocity field, and then reconnect, generating a turbulent state with only moderate density oscillations, as shown in Fig. 4(b).

We analyze the turbulent state in terms of the spatial averages of the velocity components. At the beginning of the decay, we find $\left\langle\left|v_{y}\right|\right\rangle /\left\langle\left|v_{x}\right|\right\rangle \approx 1$ and (as expected, as vortices are initially aligned in the $z$ direction) $\left\langle\left|v_{z}\right|\right\rangle /\left\langle\left|v_{x}\right|\right\rangle \approx$ 0 . Figure 5 (top) shows that, as time proceeds, the vortex configuration becomes almost isotropic; indeed at $t=10 \tau_{\mathrm{HO}}$ we have $\left\langle\left|v_{y}\right|\right\rangle /\left\langle\left|v_{x}\right|\right\rangle \approx 1.00$ and $\left\langle\left|v_{z}\right|\right\rangle /\left\langle\left|v_{x}\right|\right\rangle \approx 0.77$. For simplicity of reference, we call the resulting disordered vortex configuration a 'quasi-isotropic state'.

As opposed to the anisotropic case, the oscillations of the transverse velocity components $v_{x}$ and $v_{y}$ appear almost completely in phase for this quasi-isotropic case [compare Fig. 2 (top) and Fig. 5 (top)], and these also suggest the existence of collective modes. We have evaluated the time evolution of the condensate's transverse widths for this scenario as well. Figure 6 shows that after $t \approx 10 \tau_{\mathrm{HO}}$ there is a small $\left(\sim 0.2 \ell_{\mathrm{HO}}\right)$ in-phase oscillation of the widths (as opposed to the larger oscillations for the anisotropic case in Fig. 3). This can be identified as a breathing mode and (again, through a Fourier analysis) it was found to exhibit a characteristic frequency of $\omega \sim 2 \omega_{r}$, also in agreement with theoretical predictions for a vortex-free cloud [56]; this value is expected to hold even for rapidly rotating trapped systems [57]. These results, alongside a visual comparison of Figs. 1 and 4, show that the outer surface of the condensate is actually slightly less disturbed in the quasi-isotropic case. Our turbulent condensate [Fig. 4(b)] is clearly less 'wobbly' than condensates made 


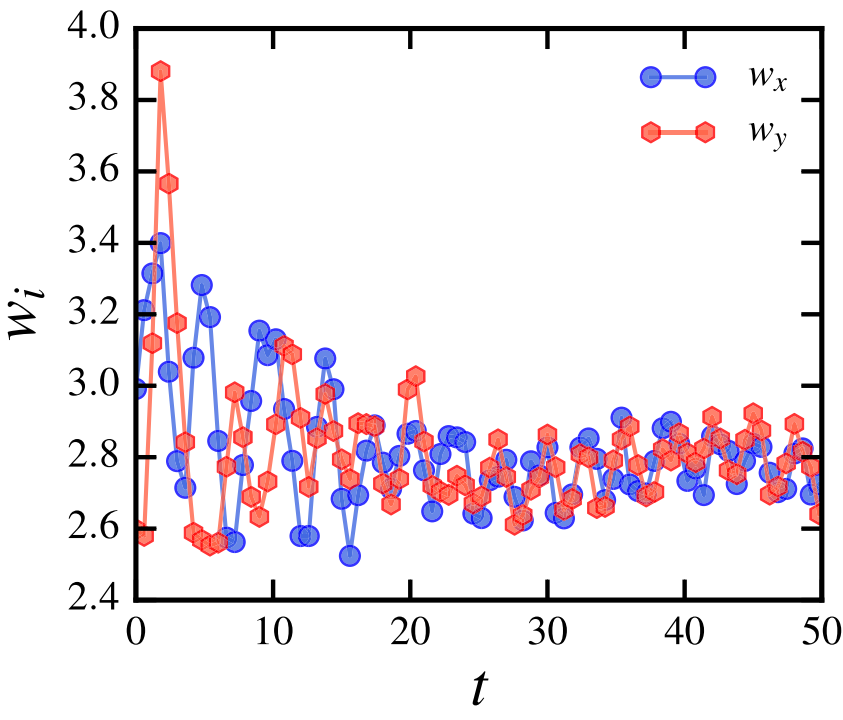

FIG. 6. Decay of antiparallel doubly charged vortices (quasiisotropic state). Time evolution of the condensate's width.

turbulent via other stirring methods, as in, notably, Refs. [29], [58], and [34]. (See the Appendix for a quantitative discussion of the surface oscillations of the condensate.)

We proceed and analyze the distribution of values of the turbulent velocity components (Fig. 7). We find that the probability density functions (PDFs, or normalized histograms) display the typical power-law scaling $\operatorname{PDF}\left(v_{i}\right) \sim v_{i}^{\alpha_{i}}$

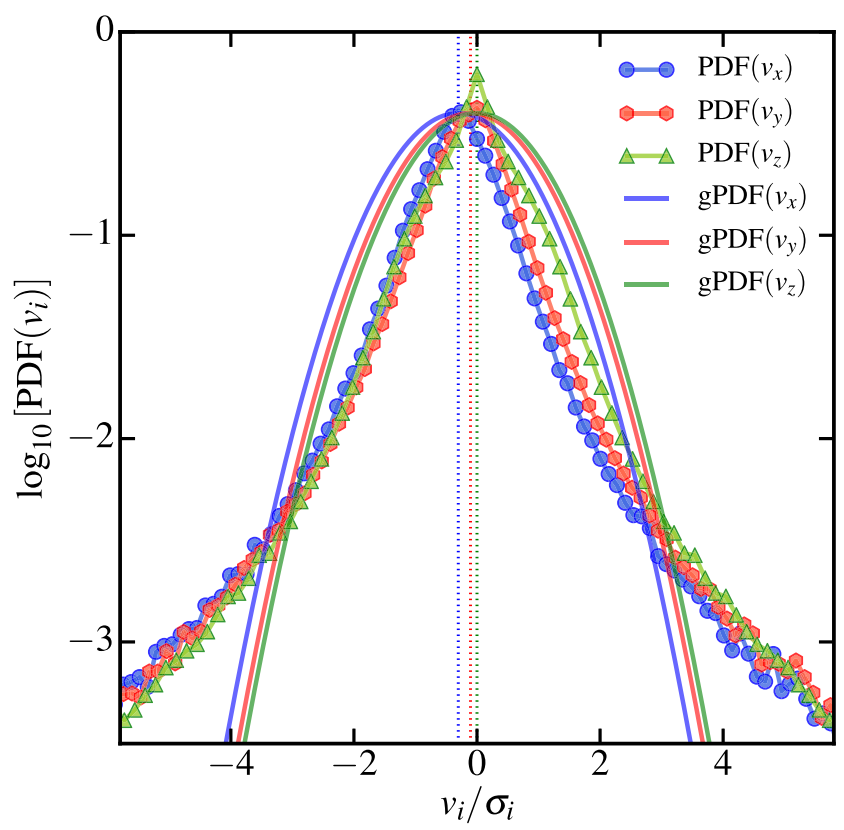

FIG. 7. Decay of antiparallel doubly charged vortices (quasiisotropic state). PDFs of velocity components $v_{i}(i=x, i=y$, and $i=z$ corresponding to blue, red, and green symbols, respectively) plotted vs $v_{i} / \sigma_{i}$ (where $\sigma_{i}$ are the corresponding standard deviations) at $t=12.6 \tau_{\mathrm{HO}}$. For reference, solid curves are Gaussian fits (gPDFs) with standard deviations $\sigma_{x}=1.8, \sigma_{y}=1.7$, and $\sigma_{z}=1.4$, and mean values (plotted as vertical lines near the origin) $\mu_{x}=-0.3$, $\mu_{y}=-0.1$, and $\mu_{z}=0.0$. $(i=x, y, z)$, where $\alpha_{x} \approx-2.97, \alpha_{y} \approx-2.95, \alpha_{z} \approx-3.20$, in agreement with findings in larger condensates [29]. Such power-law scaling, characteristic of quantum turbulence and observed in helium experiments [59], is in contrast to Gaussian PDFs, which are typical of classical turbulence. The difference between power-law and Gaussian statistics is important at high velocities (power-law PDFs have larger values in the tails) and arises from the quantization of vorticity [which creates very high velocities for $r \rightarrow 0$, as $v=\kappa /(2 \pi r)]$. For the sake of comparison, Fig. 7 also displays Gaussian fits [60].

\section{IDENTIFICATION OF THE TURBULENCE}

The two disordered vortex states, anisotropic and quasiisotropic, produced, respectively, by the decay of a single quadruply charged vortex (Sec. IV) and by the decay of two antiparallel doubly charged vortices (Sec. V), are clearly different. In the anisotropic state, all vortex lines are aligned in the same direction, and the net nonzero angular momentum constrains the flow. In the quasi-isotropic state, the oscillations of the average transverse velocity components $\left\langle v_{i}\right\rangle$ are three times larger, suggesting the presence of high-velocity events (vortex reconnections between Kelvin waves growing on opposite-oriented vortices), which are the hallmarks of turbulence. Moreover, in the quasi-isotropic state, the zero angular momentum of the initial configuration allows a redistribution of the velocity field, making the amplitudes of the three velocity components almost equal; indeed, after the initial vortex has split $\left(t \approx 10 \tau_{\mathrm{HO}}\right)$, the axial velocity, $\left\langle\left|v_{z}\right|\right\rangle \approx 0.9 \ell_{\mathrm{HO}} / \tau_{\mathrm{HO}}$, is not much lower than the transverse velocity, $\left\langle\left|v_{x}\right|\right\rangle \approx 1.2 \ell_{\mathrm{HO}} / \tau_{\mathrm{HO}}$ and $\left\langle\left|v_{y}\right|\right\rangle \approx 1.1 \ell_{\mathrm{HO}} / \tau_{\mathrm{HO}}$. On the contrary, in the anisotropic state, at the same stage $\left(t \approx 10 \tau_{\mathrm{HO}}\right)$, the axial velocity component is much smaller than the transverse components. In other words, the velocity field which results from the decay of the antiparallel doubly charged vortex state is indeed almost isotropic.

Since there is not yet a precise definition of turbulence, in principle both disordered states investigated here could be considered somewhat 'turbulent'. However, at this early stage of investigation, we want to make conceptual connections to the simple isotropic cases known in the literature (in particular, the Kolmogorov and Vinen regimes of turbulence). Therefore hereafter we concentrate on the quasi-isotropic state.

The next question is whether the quasi-isotropic state is turbulent in the sense of classical turbulence or in comparison with turbulent superfluid helium. To find the answer, we turn to the workhorse of statistical physics: the correlation function.

The quantity which measures the degree of randomness of a turbulent flow is the (normalized) longitudinal correlation functions $[9,61]$, defined by

$$
f_{i}(r)=\frac{\left\langle v_{i}\left(\mathbf{x}+r \hat{\mathbf{e}}_{i}\right) v_{i}(\mathbf{x})\right\rangle}{\left\langle v_{i}(\mathbf{x})^{2}\right\rangle},
$$

where the symbol $\langle\ldots\rangle$ denotes an average over the position vector $\mathbf{x}=(x, y, z)$, and $\hat{\mathbf{e}}_{i}$ is the unit vector in the corresponding Cartesian direction. The distance $r$ is limited by the size of the BEC, approximately the transverse and axial Thomas-Fermi radii, $d_{\mathrm{TF}}=4.2 \ell_{\mathrm{HO}}$ and $D_{\mathrm{TF}}=32.6 \ell_{\mathrm{HO}}$, respectively. From the longitudinal correlation function one 


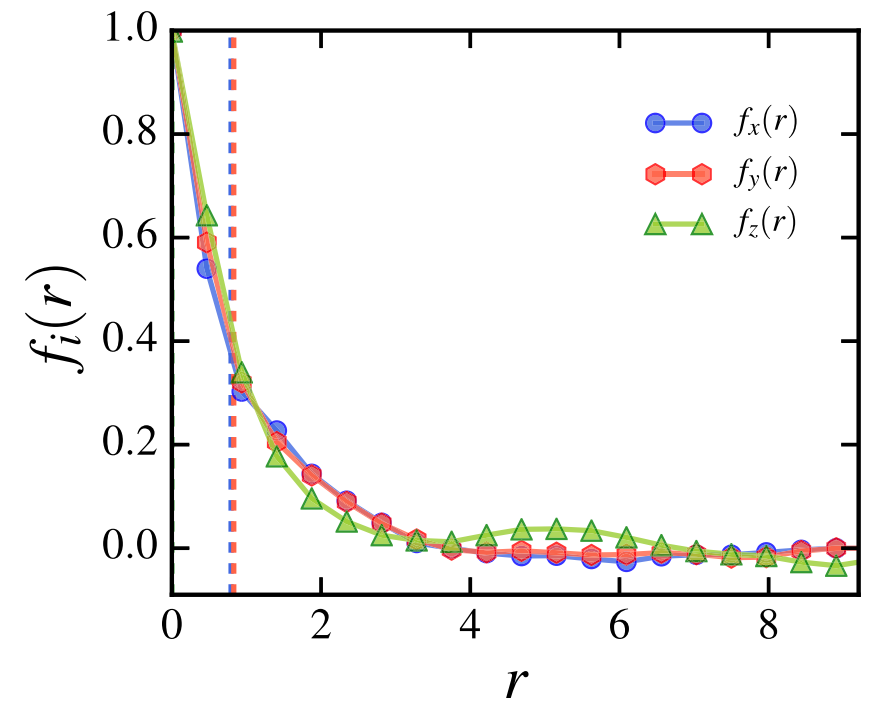

FIG. 8. Decay of antiparallel doubly charged vortices (quasiisotropic state). Longitudinal correlation functions $f_{i}(r)(i=x, y, z)$ vs $r$ (in units of $\ell_{\mathrm{HO}}$ ) at $t=13 \tau_{\mathrm{HO}}$. Dashed vertical lines indicate the length $\ell_{c}$ over which the velocity field is highly correlated, as defined in Eq. (4).

obtains the integral length scale

$$
\ell_{c}=\int_{0}^{\infty} f_{i}(r) d r .
$$

In fluid dynamics, $\ell_{c}$ represents the size of the large eddies. In our case, $\ell_{c}$ is the length scale over which the velocity field is highly correlated. Figure 8 shows that the correlation functions drop to only $\approx 10 \%$ at distances of the order of the average separation between the vortex lines, $\ell \approx 6.1 \ell_{\mathrm{HO}}$; the last quantity is estimated as $\ell \approx L^{-1 / 2}$ from the measurement of the vortex line density $L$ (the vortex length per unit volume). Physically, this lack of correlation means that the vortex lines are randomly oriented with respect to each other. The analysis of the correlation function therefore suggests that the turbulent velocity field arising from the decay of antiparallel doubly charged vortices is essentially a random flow.

This result implies that the distribution of the kinetic energy over the length scales, or energy spectrum $E(k)$ (where the wave number $k$ represents the inverse length scale), should be very different from the celebrated Kolmogorov scaling, $E(k) \sim k^{-5 / 3}$, which is observed in classical turbulence and implies a particular structure of the flow. The importance of the Kolmogorov scaling is that it is the signature of a nonlinear cascade mechanism which transfers energy from large length scales to small length scales. Besides classical turbulence, the Kolmogorov scaling has been observed in helium experiments $[62,63]$ when the turbulence is generated by grids in wind tunnels or by counter-rotating propellers. Numerical simulations [64] revealed that the Kolmogorov scaling is associated with the presence of large-scale polarization (bundles) of vortex lines which become locally parallel to each other; the bundles locally create a net average rotation over length scales larger than $\ell$, thus building up energy at wave numbers $k<k_{\ell}=2 \pi / \ell$ (the hydrodynamical range).

To compute the energy spectrum $E(k)$ of our turbulent condensate, we use a standard procedure [65] to extract

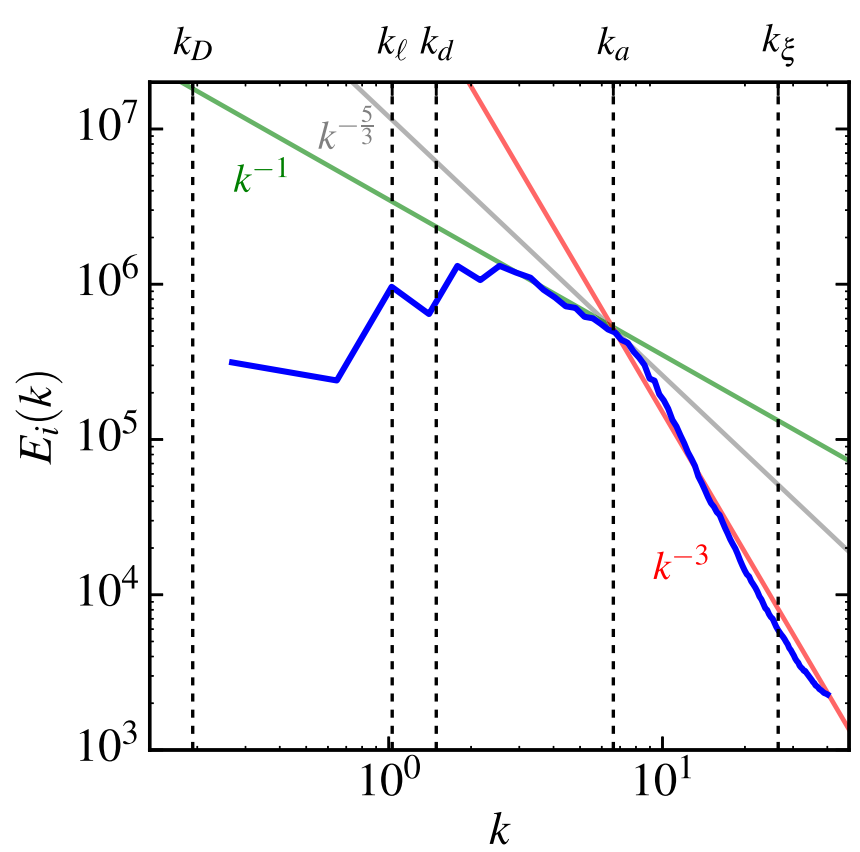

FIG. 9. Decay of two antiparallel doubly charged vortices (quasiisotropic state). Incompressible kinetic energy spectrum $E_{i}(k)$ (arbitrary units) vs wave number $k$ (in units of $\ell_{\mathrm{HO}}^{-1}$ ) at $t=12.8 \tau_{\mathrm{HO}}$. Vertical lines mark the wave numbers corresponding to the healing length $\xi=0.24 \ell_{\mathrm{HO}}$, the vortex core size $a=0.96 \ell_{\mathrm{HO}}$, the average distance between vortex lines $\ell=6.10 \ell_{\mathrm{HO}}$, and the radial and axial ThomasFermi radii $d_{\mathrm{TF}}=4.21 \ell_{\mathrm{HO}}$ and $D_{\mathrm{TF}}=32.64 \ell_{\mathrm{HO}}$. Red, gray, and green lines represent the power laws $k^{-3}, k^{-5 / 3}$, and $k^{-1}$, respectively.

the incompressible kinetic energy from the total energy, obtaining Fig. 9. To interpret the figure, we mark with vertical lines the wave numbers $k_{\xi}=2 \pi / \xi, k_{a}=2 \pi / a, k_{\ell}=2 \pi / \ell$, $k_{d}=2 \pi / d_{\mathrm{TF}}$, and $k_{D}=2 \pi / D_{\mathrm{TF}}$, corresponding to the healing length $\xi$, the vortex core radius $a$, the average vortex separation $\ell$, and the radial and axial Thomas-Fermi radii $d_{\mathrm{TF}}$ and $D_{\mathrm{TF}}$, respectively. Figure 9 shows that the energy spectrum is not of the Kolmogorov type: under the classical Kolmogorov scenario, most of the energy would be contained in the largest eddies in the small- $k$ region (the apparent deficit, estimated by extrapolating the $k^{-5 / 3}$ slope to the left, is more than two orders of magnitude). Instead of the $k^{-5 / 3}$ scaling, we observe the $E(k) \sim k^{-1}$ spectrum, which is characteristic of an isolated straight vortex line. This means that, at distances less than $\ell$, the velocity field is dominated by the nearest vortex in the vicinity of the point of observation-the effects of all the other vortices in a random tangle canceling each other out. The range of $k$ space where this scaling takes place is less than a decade because not much $k$ space is available in a typical atomic condensate (unlike superfluid helium and classical fluids), but the scaling is clearly visible (the fact that $k_{\ell}<k_{\mathrm{TF}}$ simply means that, at this time in the decay, the vortex lines are spread throughout the condensate with typical distances $\ell$ larger than the radial dimension $d_{\mathrm{TF}}$; note that $\ell<D_{\mathrm{TF}}$, of course.) It is also noteworthy that the $E(k) \sim k^{-3}$ scaling which appears in the region $k_{a}<k<k_{\xi}$ is characteristic of the vortex core $[66,67]$. We conclude that Fig. 9 is consistent with the random flow interpretation which results from the analysis of the correlation functions. 


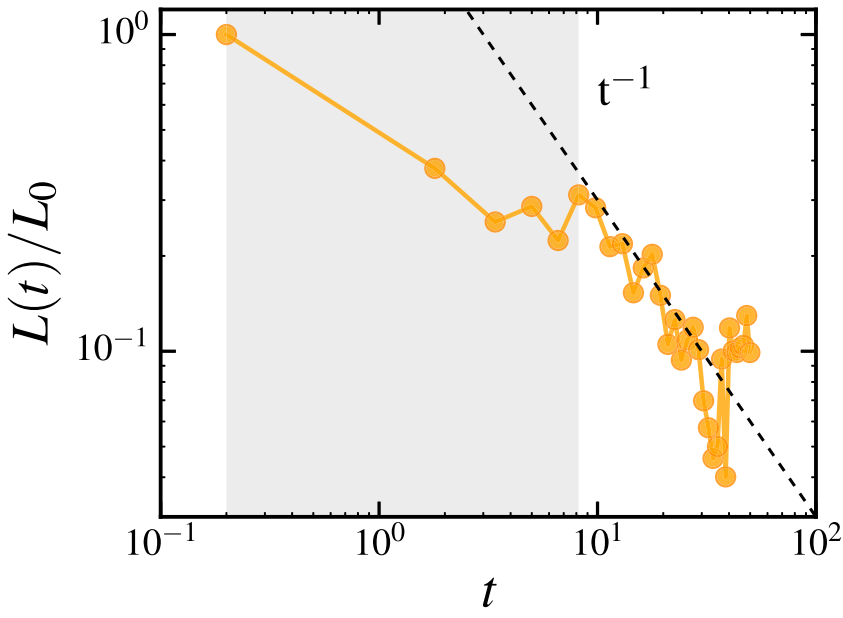

FIG. 10. Time evolution of the vortex line density ( $t$ in units of $\left.\tau_{\mathrm{HO}}\right)$. The shaded area corresponds to the time elapsed for the decay of the two antiparallel multiply charged vortices, $t \approx 8 \tau_{\mathrm{HO}}$, after which we only see singly quantized vortices in the system in the quasi-isotropic state.

Besides the correlation function and the energy spectrum, further insight into the nature of our turbulence is acquired by measuring the temporal decay of the vortex line density $L(t)$. This decay is caused by sound radiated away by vortices as they accelerate about each other [68] or reconnect [69] with each other. Figure 10 shows that, at large $t$, the decay is consistent with the form $L(t) \sim t^{-1}$, as reported for a larger spherical condensates [29]; the decaying turbulence is shown in the second movie in the Supplemental Material [51].

Therefore the short correlation length, the lack of energy at small $k$, the $E(k) \sim k^{-1}$ scaling of the energy spectrum for large $k$ in the hydrodynamical range, and the $L(t) \sim t^{-1}$ temporal behavior of the decay allow us to identify our quasiisotropic vortex state as an example of the Vinen turbulent regime in a trapped system.

\section{CONCLUSION}

In this work we have explored the decay of initially imprinted multicharged vortices as a method to generate turbulence in a trapped Bose-Einstein condensate, which is relatively free of large surface oscillations and fragmentation (see the Appendix). We have examined the decay of two multicharged vortex systems in a typical cigar-shaped, harmonically confined, atomic Bose-Einstein condensate. The first (a quadruply charged vortex) led to the disordered, anisotropic vortex state. The second (two antiparallel doubly charged vortices) generated helical Kelvin waves on oppositely oriented vortex lines which reconnected, creating a second disordered, quasi-isotropic vortex state. Looking for similarities to classical turbulence in its simplest possible forms - in particular, with the property of isotropy-we have concentrated our attention on the quasi-isotropic state and carefully considered in what sense it is turbulent.

This question is subtle. In classical physics, turbulence implies a large range of length scales which are all excited and interact nonlinearly. In classical fluids, the presence and the intensity of turbulence are inferred from the Reynolds number Re, which must be sufficiently large (typically few thousands, depending on the problem) for turbulence to exist. But the Reynolds number has two definitions. The first definition is

$$
\operatorname{Re}=\frac{u D}{v},
$$

where $D$ is the system's large length scale, i.e., the system's size or the size of the energy-containing eddies, $u$ is the flow's velocity at that large scale, and $v$ is the kinematic viscosity; this definition follows directly from the Navier-Stokes equation and measures the ratio of the magnitudes of inertial and viscous forces acting on a fluid parcel. The definition makes apparent why large-scale (e.g., geophysical) flows are always turbulent and why microfluids flows are not (indeed, with microfluids one has to rely on chaos, not turbulence, to achieve any desired mixing). The second definition assumes Kolmogorov theory and is

$$
\operatorname{Re}=\left(\frac{D}{\eta}\right)^{4 / 3}
$$

where $\eta$ is the length scale of viscous dissipation. This definition measures the degree of separation between the large length scale (at which energy is typically injected) and the small length scale (at which energy is dissipated). In the context of condensates, the two definitions clash with each other: The first definition implies that $\mathrm{Re}$ is infinite (because the viscosity is zero), and the second that Re is not much larger than unity (because the size of a typical condensate is larger, but not orders of magnitude larger, than the healing length, which can be considered the length scale at which acoustic dissipation of kinetic energy occurs). Although interesting work is in progress to identify a definition of Reynolds number suitable for a superfluid system (for example, exploring dynamical similarities [70]), to answer the question which we asked, at this stage, we have to leave the Reynolds number and proceed in other ways.

We have therefore carefully examined the properties of the disordered, quasi-isotropic state in terms of velocity statistics, energy spectrum, correlation function, and temporal decay and compared them to the properties of classical turbulence and of turbulent superfluid helium. Clearly, the quasi-isotropic state does not compare well with the properties of classical turbulence. Despite the limited range of length scales available in a small BEC, we conclude that, in the decay of the two antiparallel doubly charged vortices, the quasi-isotropy of our disordered state, the short correlation length, the properties of the energy spectrum, and the temporal behavior of the vortex decay identify our disordered, quasi-isotropic vortex state as an example of the Vinen turbulent regime first discovered in superfluid helium at low temperatures, which is interpreted as a state of turbulence without an energy cascade.

The nature of the disorder, or turbulence, in the anisotropic case generated by the decay of the single quadruply charged vortex will be the subject of future investigations: one should vary the amount of polarization and study fluctuations of the velocity field over the mean rotating flow. There are no numerical studies yet of such turbulence in trapped Bose systems, and (because of the role played by boundaries in the spin-down of viscous flows) no immediate classical analogies, so this case is less straightforward to analyze; spin-down dynamics experiments in superfluid helium [71-73] should be the main reference systems. 
In summary, our scheme describes a controllable system to investigate different regimes of quantum turbulence in trapped atomic condensates, where isotropy could be a tunable parameter (depending on the initial configuration of multicharged vortices). This control, alongside the versatility of experiments with condensates, could shed light on fundamental aspects of turbulence. Future work will, for instance, address the next natural question: Can the classical Kolmogorov regime be achieved in much larger condensates under suitable forcing at the largest length scale, as suggested by some numerical simulations [28], thus identifying the crossover between Vinen and Kolmogorov turbulence?

Finally, an important question which must be addressed by further work is whether Vinen turbulence is unique to superfluids or can be generated also in classical fluids. If the definying property of Vinen turbulence is, as it seems [5], the absence of a Kolmogorov energy cascade, the line of inquiry should start with studies of turbulent flows in wind tunnels with fractal (rather than uniform) grids, which suggest the absence of an energy cascade [74].

Data supporting this publication is openly available under an 'Open Data Commons Open Database License'. Additional metadata are available in Ref. [76]. Please contact Newcastle Research Data Service at rdm@ncl.ac.uk for access instructions.

\section{ACKNOWLEDGMENTS}

We acknowledge financial support from CAPES (PDSE Proc. No. BEX 9637/14-1), CNPq, FAPESP (program CEPID), EPSRC Grant No. EP/I019413/1, and JSPS Grant No. KAKENHI-16K05461. This research was developed making use of the computational resources (Euler cluster) of the Center for Mathematical Sciences Applied to Industry (Centro de Ciências Matemáticas Aplicadas à Indústria; CeMEAI) financed by FAPESP.

\section{APPENDIX: CHARACTERIZING SURFACE OSCILLATIONS}

In order to quantify our claim that the generation of turbulence via the decay of multicharged vortices reduces large surface oscillations of the condensate and prevents fragmentation, we introduce the following isoperimetric quotient [75] as a measure of the condensate's surface oscillations, or "wobbliness":

$$
Q \equiv \frac{4 \pi A}{P^{2}},
$$

where $P$ is the perimeter of a closed curve $C$, and $A$ the area enclosed by it. This quantity intuitively quantifies the departure from circularity. $Q$ equals its maximum value 1 for a perfect circle and reaches smaller values for greater surface oscillations of $C$.

For the time evolution of our quasi-isotropic case, we define a curve $C$ representing the edge of the condensate for a transverse cross section. An average over 40 adjacent cross sections (separated by the numerical spatial resolution) from a thin, horizontal stripe around the center of the axial $z$ axis (equivalent to less than a tenth of the condensate $z$ direction extension) was necessary to avoid numerical issues in the
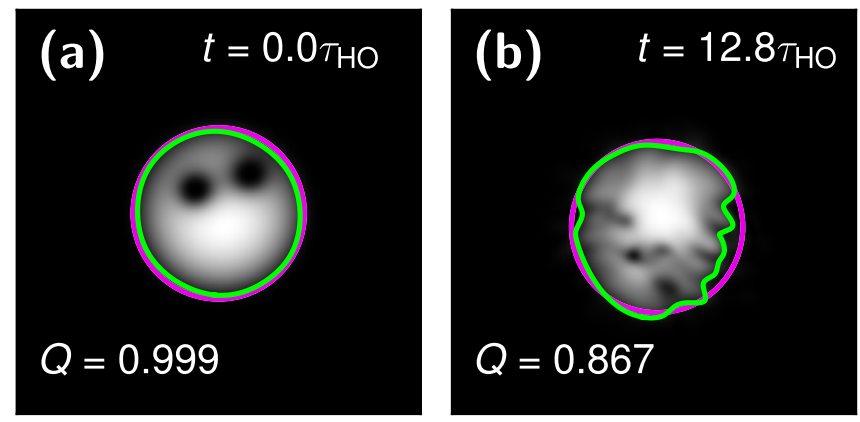

FIG. 11. Transverse cross section of the condensate in the quasiisotropic case. The green curve defines the condensate edge $C$ compared to the magenta curve, representing the radial ThomasFermi circumference for (a) the initial state and (b) at a turbulent instant. The circularity of both edges is given by $Q$ as defined in Eq. (A1).

definition of the condensate edge. See examples shown in Figs. 11(a) and 11(b) for the (green) curve $C$, compared to the radial Thomas-Fermi (magenta) circumference, for the initial state and a turbulent instant, respectively.

In Fig. 12, we see the values of $Q$ for the entire evolution. For the sake of comparison, we apply the same analysis to images taken from simulations [58] of a combined shaking and rotation of the trap that has been successfully used to generate quantum turbulence in BECs [27]. In particular, applying the criterion to images from Fig. 3 in [58], at times of 15.54 and $15.92 \mathrm{~ms}$, we find $Q=0.401$ and $Q=$ 0.386 , respectively. Their described technique clearly excites high-amplitude modes, such as scissors modes and dipolar oscillations, including other large density fluctuations, which justify such low values of circularity, compared to the induction of turbulence from the decay of multicharged vortices. We conclude that for all instants, $Q$ is much closer to 1 in our case, suggesting less perturbation of the condensate's surface. Not only does our method generate a less wobbly condensate, but also the center of mass of the cloud stays practically still, as opposed to the case in [58]. The absence of large oscillations of the center of mass in our scheme offers a practical advantage for experiments which make use of time-of-flight imaging. The less momentum the cloud's center of mass acquires, the easier to focus when taking the time-of-flight absorption image.

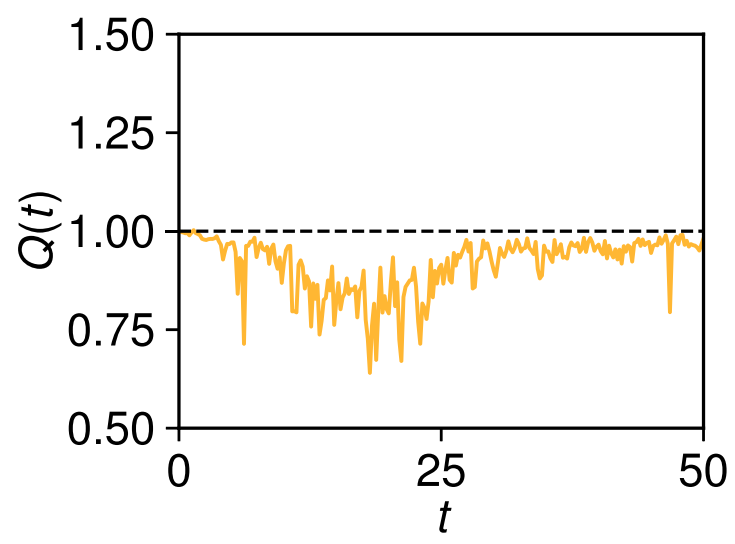

FIG. 12. Time evolution of the isoperimetric quotient ( $t$ in units of $\left.\tau_{\mathrm{HO}}\right)$. 
[1] C. F. Barenghi, V. S. L'vov, and P.-E. Roche, Proc. Natl. Acad. Sci. USA 111, 4683 (2014).

[2] U. Frisch, Turbulence: The Legacy of A. N. Kolmogorov (Cambridge University Press, Cambridge, UK, 1995).

[3] R. Hänninen and A. W. Baggaley, Proc. Natl. Acad. Sci. USA 111, 4667 (2014).

[4] P. M. Walmsley and A. I. Golov, Phys. Rev. Lett. 100, 245301 (2008).

[5] C. F. Barenghi, Y. A. Sergeev, and A. W. Baggaley, Sci. Rep. 6, 35701 (2016).

[6] A. W. Baggaley, C. F. Barenghi, and Y. A. Sergeev, Phys. Rev. B 85, 060501 (2012).

[7] W. Vinen, in Proceedings of the Royal Society of London A: Mathematical, Physical and Engineering Sciences, Vol. 240 (The Royal Society, London, 1957), pp. 114-127.

[8] A. W. Baggaley, L. K. Sherwin, C. F. Barenghi, and Y. A. Sergeev, Phys. Rev. B 86, 104501 (2012).

[9] G. W. Stagg, N. G. Parker, and C. F. Barenghi, Phys. Rev. A 94, 053632 (2016).

[10] N. G. Berloff and B. V. Svistunov, Phys. Rev. A 66, 013603 (2002).

[11] G. E. Volovik, JETP Lett. 78, 533 (2003).

[12] T. Aioi, T. Kadokura, T. Kishimoto, and H. Saito, Phys. Rev. X 1, 021003 (2011).

[13] M. C. Davis, R. Carretero-González, Z. Shi, K. J. H. Law, P. G. Kevrekidis, and B. P. Anderson, Phys. Rev. A 80, 023604 (2009).

[14] K. W. Madison, F. Chevy, W. Wohlleben, and J. Dalibard, Phys. Rev. Lett. 84, 806 (2000).

[15] C. Raman, J. R. Abo-Shaeer, J. M. Vogels, K. Xu, and W. Ketterle, Phys. Rev. Lett. 87, 210402 (2001).

[16] D. V. Freilich, D. M. Bianchi, A. M. Kaufman, T. K. Langin, and D. S. Hall, Science 329, 1182 (2010).

[17] M. C. Tsatsos, P. E. Tavares, A. Cidrim, A. R. Fritsch, M. A. Caracanhas, F. E. A. dos Santos, C. F. Barenghi, and V. S. Bagnato, Phys. Rep. 622, 1 (2016).

[18] C. F. Barenghi, L. Skrbek, and K. R. Sreenivasan, Proc. Natl. Acad. Sci. USA 111, 4647 (2014).

[19] L. Skrbek and K. R. Sreenivasan, Phys. Fluids 24, 011301 (2012).

[20] T. W. Neely, E. C. Samson, A. S. Bradley, M. J. Davis, and B. P. Anderson, Phys. Rev. Lett. 104, 160401 (2010).

[21] A. C. White, C. F. Barenghi, and N. P. Proukakis, Phys. Rev. A 86, 013635 (2012).

[22] A. C. White, N. P. Proukakis, and C. F. Barenghi, J. Phys.: Conf. Ser. 544, 012021 (2014).

[23] W. J. Kwon, G. Moon, J. Y. Choi, S. W. Seo, and Y. I. Shin, Phys. Rev. A 90, 063627 (2014).

[24] G. W. Stagg, A. J. Allen, N. G. Parker, and C. F. Barenghi, Phys. Rev. A 91, 013612 (2015).

[25] A. J. Allen, N. G. Parker, N. P. Proukakis, and C. F. Barenghi, Phys. Rev. A 89, 025602 (2014).

[26] A. Cidrim, F. E. A. dos Santos, L. Galantucci, V. S. Bagnato, and C. F. Barenghi, Phys. Rev. A 93, 033651 (2016).

[27] E. A. L. Henn, J. A. Seman, G. Roati, K. M. F. Magalhães, and V. S. Bagnato, Phys. Rev. Lett. 103, 045301 (2009).

[28] M. Kobayashi and M. Tsubota, Phys. Rev. A 76, 045603 (2007).

[29] A. C. White, C. F. Barenghi, N. P. Proukakis, A. J. Youd, and D. H. Wacks, Phys. Rev. Lett. 104, 075301 (2010).
[30] C. N. Weiler, T. W. Neely, D. R. Scherer, A. S. Bradley, M. J. Davis, and B. P. Anderson, Nature 455, 948 (2008).

[31] L. Chomaz, L. Corman, T. Bienaimé, R. Desbuquois, C. Weitenberg, S. Nascimbène, J. Beugnon, and J. Dalibard, Nat. Commun. 6, 6162 (2015).

[32] G. Lamporesi, S. Donadello, S. Serafini, F. Dalfovo, and G. Ferrari, Nat. Phys. 9, 656 (2013).

[33] N. Navon, A. L. Gaunt, R. P. Smith, and Z. Hadzibabic, Science 347, 167 (2015).

[34] N. G. Parker and C. S. Adams, Phys. Rev. Lett. 95, 145301 (2005).

[35] C. Barenghi and N. Parker, A Primer on Quantum Fluids, Springer Briefs in Physics (Springer International, Cham, Switzerland, 2016).

[36] Y. Shin, M. Saba, M. Vengalattore, T. A. Pasquini, C. Sanner, A. E. Leanhardt, M. Prentiss, D. E. Pritchard, and W. Ketterle, Phys. Rev. Lett. 93, 160406 (2004).

[37] M. Kumakura, T. Hirotani, M. Okano, Y. Takahashi, and T. Yabuzaki, Phys. Rev. A 73, 063605 (2006).

[38] M. Okano, H. Yasuda, K. Kasa, M. Kumakura, and Y. Takahashi, J. Low Temp. Phys. 148, 447 (2007).

[39] T. Isoshima, M. Okano, H. Yasuda, K. Kasa, J. A. M. Huhtamäki, M. Kumakura, and Y. Takahashi, Phys. Rev. Lett. 99, 200403 (2007).

[40] H. Pu, C. K. Law, J. H. Eberly, and N. P. Bigelow, Phys. Rev. A 59, 1533 (1999).

[41] M. Möttönen, T. Mizushima, T. Isoshima, M. M. Salomaa, and K. Machida, Phys. Rev. A 68, 023611 (2003).

[42] J. A. M. Huhtamäki, M. Möttönen, and S. M. M. Virtanen, Phys. Rev. A 74, 063619 (2006).

[43] J. A. M. Huhtamäki, M. Möttönen, T. Isoshima, V. Pietilä, and S. M. M. Virtanen, Phys. Rev. Lett. 97, 110406 (2006).

[44] A. M. Mateo and V. Delgado, Phys. Rev. Lett. 97, 180409 (2006)

[45] T. Kuwamoto, H. Usuda, S. Tojo, and T. Hirano, J. Phys. Soc. Jpn. 79, 034004 (2010).

[46] P. Kuopanportti and M. Möttönen, Phys. Rev. A 81, 033627 (2010)

[47] M. Nakahara, T. Isoshima, K. Machida, S.-i. Ogawa, and T. Ohmi, Physica B: Condens. Matter 284-288, 17 (2000).

[48] A. E. Leanhardt, A. Görlitz, A. P. Chikkatur, D. Kielpinski, Y. Shin, D. E. Pritchard, and W. Ketterle, Phys. Rev. Lett. 89, 190403 (2002).

[49] Y. Kawaguchi and T. Ohmi, Phys. Rev. A 70, 043610 (2004).

[50] G. R. Dennis, J. J. Hope, and M. T. Johnsson, Comput. Phys. Commun. 184, 201 (2013).

[51] See Supplemental Material at http://link.aps.org/supplemental/ 10.1103/PhysRevA.96.023617. Movie 1: real decay of a $j=4$ multicharged vortex line. Movie 2: real decay of two antiparallel, doubly charged vortex lines.

[52] E. Fonda, D. P. Meichle, N. T. Ouellette, S. Hormoz, and D. P. Lathrop, Proc. Natl. Acad. Sci. USA 111, 4707 (2014).

[53] V. Bretin, P. Rosenbusch, F. Chevy, G. V. Shlyapnikov, and J. Dalibard, Phys. Rev. Lett. 90, 100403 (2003).

[54] N. L. Smith, W. H. Heathcote, J. M. Krueger, and C. J. Foot, Phys. Rev. Lett. 93, 080406 (2004).

[55] T. P. Simula, Phys. Rev. A 84, 021603 (2011).

[56] S. Stringari, Phys. Rev. Lett. 77, 2360 (1996).

[57] G. Watanabe, Phys. Rev. A 73, 013616 (2006). 
[58] J. A. Seman, E. A. L. Henn, R. F. Shiozaki, G. Roati, F. J. Poveda-Cuevas, K. M. F. Magalhães, V. I. Yukalov, M. Tsubota, M. Kobayashi, K. Kasamatsu et al., Laser Phys. Lett. 8, 691 (2011).

[59] M. S. Paoletti, M. E. Fisher, K. R. Sreenivasan, and D. P. Lathrop, Phys. Rev. Lett. 101, 154501 (2008).

[60] The definition is $\operatorname{gPDF}\left(v_{i}\right)=\left(2 \pi \sigma_{i}^{2}\right)^{-1 / 2} \exp \left[-\left(v_{i}-\mu_{i}\right)^{2} /\right.$ $\left.\left(2 \sigma_{i}^{2}\right)\right]$, where $\mu_{i}$ and $\sigma_{i}(i=x, y, z)$ are the mean values and the standard deviations, respectively.

[61] P. Davidson, Turbulence: An Introduction for Scientists and Engineers (Oxford University Press, Oxford, UK, 2004).

[62] J. Maurer and P. Tabeling, Europhys. Lett. 43, 29 (1998).

[63] J. Salort, C. Baudet, B. Castaing, B. Chabaud, F. Daviaud, T. Didelot, P. Diribarne, B. Dubrulle, Y. Gagne, F. Gauthier, A. Girard, B. Hébral, B. Rousset, P. Thibault, and P.-E. Roche, Phys. Fluids 22, 125102 (2010).

[64] A. W. Baggaley, J. Laurie, and C. F. Barenghi, Phys. Rev. Lett. 109, 205304 (2012).

[65] C. Nore, M. Abid, and M. E. Brachet, Phys. Rev. Lett. 78, 3896 (1997).

[66] G. Krstulovic and M. Brachet, Phys. Rev. Lett. 105, 129401 (2010).
[67] A. S. Bradley and B. P. Anderson, Phys. Rev. X 2, 041001 (2012).

[68] M. Leadbeater, D. C. Samuels, C. F. Barenghi, and C. S. Adams, Phys. Rev. A 67, 015601 (2003).

[69] M. Leadbeater, T. Winiecki, D. C. Samuels, C. F. Barenghi, and C. S. Adams, Phys. Rev. Lett. 86, 1410 (2001).

[70] M. T. Reeves, T. P. Billam, B. P. Anderson, and A. S. Bradley, Phys. Rev. Lett. 114, 155302 (2015).

[71] P. Walmsley, A. Golov, H. Hall, W. Vinen, and A. Levchenko, J. Low Temp. Phys. 153, 127 (2008).

[72] J. Hosio, V. Eltsov, P. Heikkinen, R. Hänninen, M. Krusius, and V. L'vov, Nat. Commun. 4, 1614 (2013).

[73] J. J. Hosio, V. B. Eltsov, M. Krusius, and J. T. Mäkinen, Phys. Rev. B 85, 224526 (2012).

[74] N. Mazellier and J. C. Vassilicos, Phys. Fluids 22, 075101 (2010).

[75] H. T. Croft, K. Falconer, and R. K. Guy, Unsolved Problems in Geometry: Unsolved Problems in Intuitive Mathematics, Vol. 2 (Springer Science \& Business Media, Berlin, 2012).

[76] A. Cidrim, A. White, A. J. Allen, V. Bagnato, and C. Barenghi, Numerical data from mean-field simulations of a trapped atomic superfluid in a Vinen turbulent regime, Newcastle University, 2017, doi:10.17634/101785-6. 